\title{
Macintosh laryngoscope vs. Pentax-AWS video laryngoscope: comparison of efficacy and cardiovascular responses to tracheal intubation in major burn patients
}

\author{
Chul-Ho Woo ${ }^{1}$, Sung Hoon Kim ${ }^{1}$, Jae-Young Park ${ }^{1}$, Ji Young Bae ${ }^{1}$, In-Suk Kwak ${ }^{1}$, Sung Ha Mun², and \\ Kwang-Min $\mathrm{Kim}^{1}$ \\ Department of Anesthesiology and Pain Medicine, ${ }^{1}$ Hangang Sacred Heart Hospital, College of Medicine, Hallym University, \\ ${ }^{2}$ Kang Buk Samsung Hospital, College of Medicine, Sungkyunkwan University, Seoul, Korea
}

Background: Patients with major burns accompanied with airway edema need more attention for airway management. Although the Pentax-AWS has an advantage in managing endotracheal intubation more easily, its usefulness cannot be assured if it does not maintain hemodynamic stability in burn patients. The aim of this study was to compare cardiovascular responses and general efficacy of the Pentax-AWS and Macintosh laryngoscopes in burn patients.

Methods: American Society of Anesthesiologists physical status 2 or 3 adult patients with major burn injury were randomly assigned to group $P($ AWS, $n=50$ ) or group $M($ Macintosh, $n=50$ ). Fifty-nine patients assigned to the Macintosh group and no patient to AWS group were excluded because of failure to first intubation. Hemodynamic data at baseline, just before and after intubation as well as 3, 5 and 10 minutes after intubation and grade of sore throat were recorded in two groups. Intubation time, success rate of intubation, number of intubation attempts and the percentage of glottic opening (POGO) scores were also observed and analyzed in all patients.

Results: A statistically significant increase in heart rate just after laryngoscopy was seen in group $\mathrm{M}$. The success rate of the first attempt in group P (100\%) was greater than with the group M (46\%). POGO scores were higher in group P (97 $\pm 4 \%)$ than in group $\mathrm{M}(48 \pm 29 \%)$

Conclusions: The use of Pentax-AWS offers a reduced degree of heart rate elevation compared with the Macintosh laryngoscope and better intubation environments in major burn patients. (Korean J Anesthesiol 2012; 62: 119-124)

Key Words: Burns, Hemodynamics, Intratracheal, Intubation, Laryngoscopes.

Received: May 2, 2011. Revised: 1st, May 31, 2011; 2nd, June 28, 2011. Accepted: June 29, 2011.

Corresponding author: Ji Young Bae, M.D., Department of Anesthesiology and Pain Medicine, Hangang Sacred Heart Hospital, College of Medicine, Hallym University, 94-200, Yeongdeungpo-dong, Yeongdeungpo-gu, Seoul 150-719, Korea. Tel: 82-2-2639-5505, Fax: 82-2-2631-4387, E-mail: baejjy@hanmail.net

(c) This is an open-access article distributed under the terms of the Creative Commons Attribution Non-Commercial License (http:// creativecommons.org/licenses/by-nc/3.0/), which permits unrestricted non-commercial use, distribution, and reproduction in any medium, provided the original work is properly cited. 


\section{Introduction}

Accurate and safe endotracheal intubation is essential in medical conditions that need mechanical ventilation or general anesthesia. Failure of airway management may result in fatalityfrom brain damage or cardiopulmonary arrest in addition to other issues including tooth damage or airway injury [1]. In particular, patients with burns over $25 \%$ of their total body surface area are likely to have facial burns as well as inhalation injury due to smoke or flame from the fire, resulting in difficult intubation. Even patients without burns on the face and the neck may receive large amounts of fluids in the early stages of burn treatment; this can cause secondary generalized and oral cavity/airway edema which also adds to a more difficult airway management [2].

Various types of video laryngoscopes are useful in difficult airway management, among which Pentax-AWS (AWS) (Pentax-AWS ${ }^{\circledR}$, Pentax, Japan) has been recently developed. Its transparent blade (P-blade ${ }^{\circledR}$, Pentax, Japan) has the separated space for a $12 \mathrm{~cm}$ cable for a camera and a suction catheter, and a tracheal tube that can be attached to the right side of the blade. Also, a camera tube and a 2.4-inch LCD monitor are attached to the main unit which is connected to the blade.

Different from Macintosh laryngoscope that pressures the epiglottic vallecula, AWS lifts the epiglottis to confirm the vocal cord location and performs endotracheal intubation. It has been reported that laryngeal findings can be improved during endotracheal intubation when using AWS compared to Macintosh laryngoscope [3-5]. Some studies reported no significant differences in cardiovascular changes during endotracheal intubation $[6,7]$, while another group reported less cardiovascular changes with AWS compared to the Macintosh laryngoscope [8].

Excessive hemodynamic changes can be fatal in severe burn patients with a hypermetabolic state characterized by changes in blood flow, capillary permeability, protein synthesis and cardiac output in addition to accompanying tachycardia [2]. Therefore, it is necessary to examine the hemodynamic differences during endotracheal intubation when using AWS compared to Macintosh laryngoscope in severe burn patients.

Thus, the authors compared the efficacy and hemodynamic changes during the endotracheal intubation in severe burn patients between the AWS and Macintosh laryngoscopes.

\section{Materials and Methods}

This study was approved by the Ethics Committee of the hospital as well as the subjects or their caretakers after the study objectives were described prior to the operation.

One hundred-fifty nine patients, aged $18-65$, scheduled for regular escharectomy under general anesthesia with a hypermetabolic state due to burn injury (occurring less than one month from surgery) with a American Society of Anesthesiologists (ASA) physical status of 2 and 3 and who had second or third degree burns over $25 \%$ of body surface were selected. Patients with loose teeth, craniocervical or cervical injury,or malformation, arteriosclerosis, uncontrolled hypertension, myocardial infarction, cerebrovascular disease, class 4 of Mallampati, or existing endotracheal intubation were excluded. Also, patients with bandages due to burns in the face or neck and with difficulties in manual ventilation were excluded.

At 30 minutes before arriving at the operating room, an intramuscular injection of $0.2 \mathrm{mg}$ glycopyrrolate was given to the subjects and then Mallampati classification and the thyromental distance were measured after the arrival. The subjects were divided into either the AWS (Group P) or Macintosh group (Group M) by simple random sampling with 50 subjects each group. An electrocardiogram, automated noninvasive blood pressure monitor, and pulse oximeter were placed to measure the vital signs. Heart rate, systolic blood pressure, and diastolic blood pressure just before the anesthesia were measured and then a $10-\mathrm{cm}$ pillow was placed under the atlanto-occipital joint and preoxygenation with $100 \% \mathrm{O}_{2}$ was performed at $8 \mathrm{~L} /$ min for 3 minutes. Then, anesthesia was induced with $1.5 \mathrm{mg} /$ $\mathrm{kg}$ of propofol and unconsciousness was confirmed by the eyelid reflex test, rocuronium $(0.8 \mathrm{mg} / \mathrm{kg})$ was injected and muscle relaxation was confirmed by the loss of TOF. In group $\mathrm{P}$, a tracheal tube was previously installed in the AWS; in group M, a laryngoscope with a Macintosh \#3 curved blade was used in females and with a \#4 curved blade in males (TC-5111, Solco Biomedical Co., Pyeongtaek, Korea). Endotracheal intubation was done in both groups and the inner diameter of the tube was $7 \mathrm{~mm}$ for females and $8 \mathrm{~mm}$ for males $\left(\mathrm{Hi}-\mathrm{Lo}^{\mathrm{TM}}\right.$, Mallinckrodt Medical, Athlone, Ireland).

The time taken for endotracheal intubation as well as the POGO (percentage of glottic opening) score were recorded [9]. POGO score is the degree of glottic view after inserting a laryngoscope and expressed as a percentage; it is expressed as $100 \%$ for a full view of the glottis and $0 \%$ for no observable glottis, and measured in units of $10 \%$. The time taken for endotracheal intubation was recorded by an observer from the moment when the blade of the laryngoscope passed the incisor to the moment when it was outside the oral cavity after endotracheal intubation. The POGO score was based on subjectivity of the operator. Failure of intubation was defined as unsuccessful intubationin the trachea within 30 seconds for the first attempt; in case of failure of the first attempt, the second attempt was performed after manual ventilation with $100 \%$ oxygen for 30 seconds. After the second attempt, cricoids pressure was applied in Group P and cricoids pressure and 
a stylet were used in Group M. Also, in case of failure of both attempts, endotracheal intubation using a laryngeal mask airway or a fiberoptic bronchoscope was be performed. Patients in whom there was failure to intubate on the first attempt were excluded from hemodynamic analysis.

The anesthesia was maintained with $\mathrm{O}_{2}$ at $1.5 \mathrm{~L} / \mathrm{min}, \mathrm{N}_{2} \mathrm{O}$ at $1.5 \mathrm{~L} / \mathrm{min}$ and sevoflurane at 2 vol\%; tidal volume was $10 \mathrm{ml} / \mathrm{kg}$ and the respiratory rate was 12 . All endotracheal intubations were performed by a resident in the Department of Anesthesiology \& Pain Medicine, with over 3 years of experience in endotracheal intubation using the Macintosh laryngoscope and with more than 50 procedures using the Pentax-AWS. Heart rate, systolic blood pressure and diastolic blood pressure were measured just after intubation and at 3, 5, and 10 minutes after intubation. At 24 hours after the operation, the presence and degree of sore throat was evaluated in patients who could talk in the ward or intensive care unit. Sore throat was evaluated using a 4 grade scale $(0-3)$ defined as follows: no sore throat as 0 grade, complaints of sore throat only on asking as 1 grade, complaints of sore throat on his/her own as 2 grade, and change of voice or hoarseness, associated with throat pain as 4 grade [8].

A power analysis was performed to determine the number of patients using the results of the previous study [8]. By calculating $95 \%$ power $(1-\beta)$ and $0.01 \alpha$ value, the minimum number of patients was 48 and thus 50 was decided as the number of patients in each group. When the first intubation attempt failed, it was not included either group and the hemodynamic data was not analyzed. Enrollment continued until 50 patients per group was achieved.

Statistical analysis was performed by using SPSS 13.0 (SPSS 13.0 for Windows, IBM Corporation, USA) and intergroup demographic data including age, height, body weight, thyromental distance and total body surface area were compared using t-test. Sex, ASA physical status classification

Table 1. Patients' Characteristics and Clinical Data

\begin{tabular}{lcc}
\hline & Group M ( $\mathrm{n}=50)$ & Group P ( $=50)$ \\
\hline Age (yr) & $47.4 \pm 10.5$ & $45.5 \pm 10.4$ \\
Body weight (kg) & $65.9 \pm 11.5$ & $66.6 \pm 16.0$ \\
Height (cm) & $166.4 \pm 9.6$ & $167.0 \pm 9.3$ \\
Sex (M/F) & $38 / 12$ & $37 / 13$ \\
ASA class (II/III) & $37 / 13$ & $34 / 16$ \\
Mallampati class (I/II/III) & $6 / 29 / 15$ & $8 / 32 / 10$ \\
Thyromental distance (cm) & $7.8 \pm 0.5$ & $7.6 \pm 0.3$ \\
Percent TBSA of burn (\%) & $35.8 \pm 5.5$ & $36.2 \pm 5.6$ \\
Sore throat (0/1/2/3) & $24 / 20 / 6 / 0$ & $21 / 24 / 5 / 0$ \\
\hline
\end{tabular}

Values are means \pm SD or number of patients. There was no significant differences between groups. Group M: Macintosh group, Group P: Pentax-AWS group, TBSA: total body surface area. Sore throat scale 0 : no sore throat, 1: complaints of sore throat only on asking, 2: complaints of sore throat on his/her own, 3: change of voice or hoarseness associated with throat pain. and the presence and degree of sore throat were compared using a Chi-square test. For systolic and diastolic blood pressures and heart rate, the overall difference between groups and intra-group comparison were evaluated using repeated measures ANOVA, and a contrast test was performed for time range comparisons for the initial value in the group. POGO scores and time for intubation between two groups were compared using a t-test. The success rate of the first attempt was calculated by the ratio of the number of patients successful in the first attempt to the total number of patients using two laryngoscopes and compared by a Chi-square test. $\mathrm{P}$ values less than 0.05 were considered statistically significant.

\section{Results}

There were no differences in gender, age, height, body weight, ASA physical status classification, Mallampati class distribution, thyrometal distance, range of burn injury, and the presence and the degree of sore throat 24 hours after operation between Group M and Group P (Table 1). The number of patients with over 2nd degree of burn in the face and the neck was $35(70 \%)$ in Group M and 37 (74\%) in Group P, with no significant differences between the groups. Among 59 patients whose intubation failed in the first attempt with the Macintosh laryngoscope, the number of patients with burn in the face and neck was 43 (73\%). Among 109 patients who randomized for the Macintosh laryngoscope, $78(72 \%)$ had burn in the face and the neck but this was not significantly different compared to Group P. Among all patients designated in the Macintosh laryngoscope, the number of patients with burn in the face and neck was not statistically different between Group M and 59 patients who failed in the first attempt.

The success rate for the first attempt of endotracheal intubation was $100 \%$ for patients in Group P. Among the 109 patients designated for intubation with theMacintosh, only 50 patients had a successful first attempt was classified as Group $M$ (success rate of first attempt, 46\%). In the 59 patients with failure of first attempt, the second attempt was successful by using cricoids pressure and a stylet. The POGO score was significantly higher in Group P compared to Group M (Table 2). The POGO score for the 59 patients who underwent a second

Table 2. Intubation Profiles

\begin{tabular}{lcc}
\hline & Group M & Group P \\
\hline Patient number & 50 & 50 \\
POGO score (\%) & $48 \pm 29 *$ & $97 \pm 4$ \\
Successful intubation time (s) & $15 \pm 2$ & $15 \pm 2$ \\
\hline
\end{tabular}

Values are means \pm SD. Group M: Macintosh laryngoscope group, Group P: Pentax-AWS group, POGO: percentage of glottic opening. $* \mathrm{P}<0.01$ compared to Group $\mathrm{P}$. 


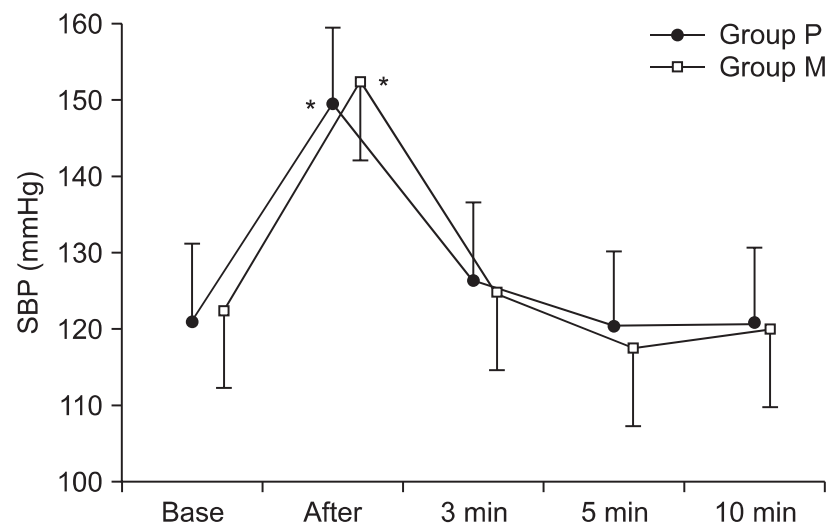

Fig. 1. Changes in systolic blood pressure (SBP) (means \pm standard deviation). Group P: Pentax-AWS, Group M: Macintosh laryngoscope. Base: before induction value. After: just after intubation value. 3, 5,10 min: value at $3,5,10$ minutes after intubation. There were no significant differences between groups. $* \mathrm{P}<0.05$ compared to baseline.

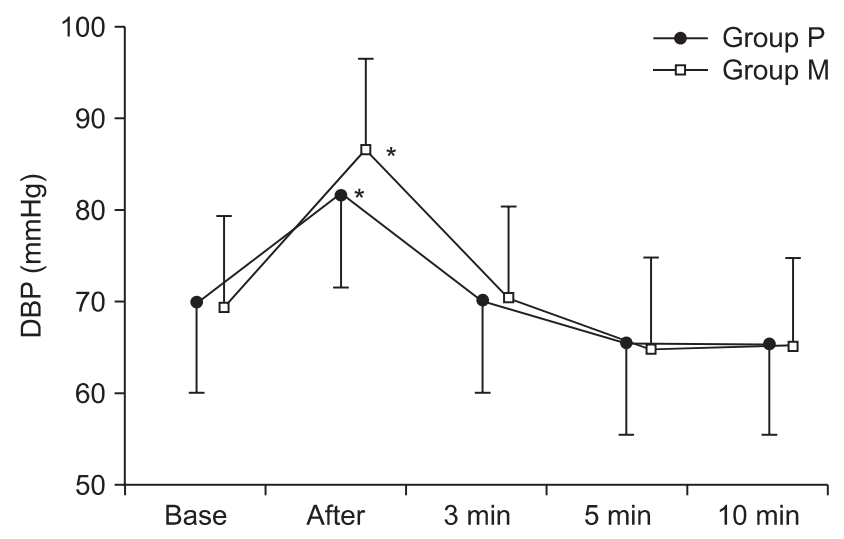

Fig. 2. The changes of diastolic blood pressure (DBP) (means \pm standard deviation). Group P: Pentax-AWS, Group M: Macintosh laryngoscope. Base: before induction value. After: just after intubation value. 3, 5, 10 min: value at $3,5,10$ minutes after intubation. There were no significant differences between groups. ${ }^{*} \mathrm{P}<0.05$ compared to base.

attempt with the Macintosh laryngoscope was $88 \pm 5 \%$, which was significantly lower compared to Group P. The time taken for intubation was not different between Group M and Group P, but the time taken for successful second intubation in patients who failed in the first attempt with the Macintosh laryngoscope was $64 \pm 46$ seconds, which was significantly longer compared to Group P.

Systolic and diastolic pressures between the two groups were not significantly different just before and after endotracheal intubation as well as at 3, 5, and 10 minutes after endotracheal intubation, but heart rate was significantly increased right after intubation in Group M compared to Group P. Systolic and diastolic pressures and heart rate were significantly increased in

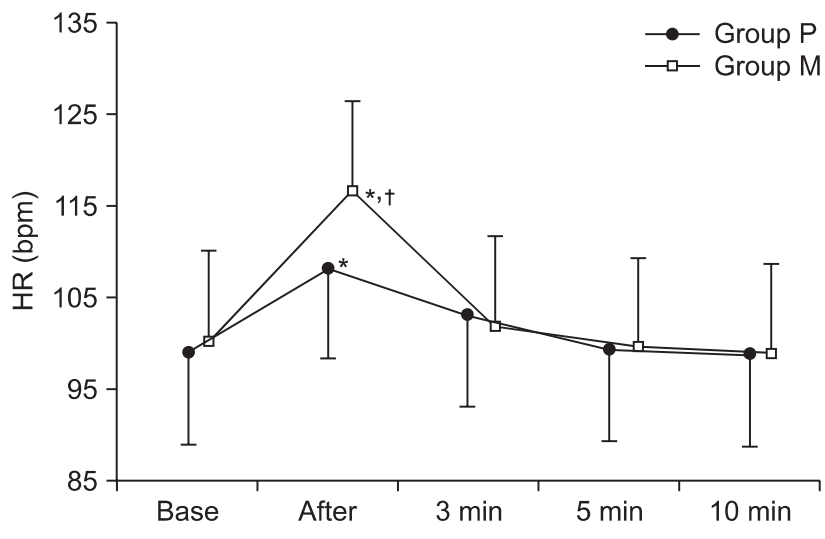

Fig. 3. Changes in heart rate (HR) (means \pm standard deviation). Group P: Pentax-AWS, Group M: Macintosh laryngoscope. Base: before induction value. After: just after intubation value. 3, 5, 10 min: value at $3,5,10$ minutes after intubation. A statistically significant difference in heart rate between groups was seen just after intubation. ${ }^{*} \mathrm{P}<0.05$ compared to base. ${ }^{\dagger} \mathrm{P}<0.05$ compared to group $P$.

both groups after intubation compared to just before the intubation, but not significantly different after 3 minutes (Fig. 1-3).

No critical complications related to anesthesia and airway management were observed in patients who participated in the study.

\section{Discussion}

This study showed that the use of AWS made endotracheal intubation faster and easier in severe burn patients and they had less increased heart rate just after the intubation compared to the Macintosh laryngoscope group.

Difficulty with endotracheal intubation is an unsolved problem for anesthesiologists and the probability of failure significantly increases in severe burn patients with accompanying facial burns, airway edema and limited cervical movement due to pain [10]. In this study with burn patients, the ratio of patients with Class 3 Mallampati classification, one of the indexes for predicting difficult endotracheal intubation, was $25 \%$ of the total subjects. Compared with a similar domestic study with non-burn patients [6] in which the ratio of patients with Class 3 Mallampati classification was $2 \%$, it was confirmed that burn patients in this study had oral cavity edema and the mouth opening was not sufficient due to pain or contracture.

Macintosh laryngoscope is still the most used tool in endotracheal intubation but cases of difficult endotracheal intubation such as Cormack and Lehane grade 3 and 4 have been reported to have success rates as low as $10-11 \%[11,12]$. Repeated attempts of intubation under difficult conditions with no view of the larynx can cause serious damage to soft tissues $[13,14]$. Thus, visual techniques, which can aid intubation while 
directly confirming the advance of the tube or the anatomical structure during difficult endotracheal intubation, have been recently highlighted [15]. Many video laryngoscopes have been developed and the Glidescope (Glidescope ${ }^{\circledR}$, Saturn Biomedical Systems Inc., Canada) and Airtraq (Airtraq ${ }^{\circledR}$, Prodol Meditec, Spain) were first commercialized. Glidescope confirms the location of the epiglottis and glottis with a separate monitor by attaching a camera at the tip of a curved blade, and endotracheal intubation can be done by inserting a properly bent stylet into a tracheal tube. The Airtraq, in which a tracheal tube can be attached to the blade, is structurally similar to the AWS but is a tool confirming the glottis through a lens at the end of the handle. Both of them are similarto the Macintish laryngoscope in which the tip of the blades enter the epiglottic vallecula and indirectly lifts the epiglottis. Compared to the AWS, it is easy to bump into the arytenoid cartilage or the epiglottis when the tracheal tube advances, and the distance between the tip of the blade and the tip of the tracheal tube is farther than with the AWS, making it difficult for tracheal tube manipulation [16].

Because of of the AWS design, the POGO score for Group P was $97 \%$, even under difficult conditions presented by severe burn patients with frequently accompanied edema of the face and oral cavity; indeed, the success rate was significantly higher compared to use of the Macintosh laryngoscope. In addition, as the average POGO score was $88 \%$ in non-burned patients in another study [3], AWS can provide sufficient glottis exposure even in severe burn patients with higher possibility of epiglottic and glottis edema, which is very important in increasing the success rate of endotracheal intubation. Unfortunately, patients with existing endotracheal intubation were excluded in the study. Because patients with expected severe airway edema are intubated endotracheally immediately after hospitalization, they were not included in the study. If a study on these patients was performed, the advantage of AWS for difficult intubation would likely be more clearly contrasted.

Hemodynamic changes due to endotracheal intubation need special attention particularly in patients with hypertension or cerebrovascular disease, and also in severe burn patients. Thus, not only improving the view of the larynx but also the minimization of hemodynamic changes is an important issue when using laryngoscopes with monitors. In this study, heart rate and blood pressure measured before inducing anesthesia and 3 minutes after the intubation were not different between the two groups, but the increase in heart rate was significantly lower right after the intubation in Group P compared to Group $\mathrm{M}$. This was thought to develope because of the difference in force applied to the oropharynx during the laryngoscope examination. Two major factors that can cause hemodynamic reactions during endotracheal intubation are the stimulation of the oropharynx by laryngoscopic examination and the stimulation to the larynx and trachea due to endotracheal intubation of the tube [17]. The maximum force applied to the base of the tongue during the laryngoscopic examination using a Macintosh laryngoscope is about 4-5 kg [17] while the AWS does not requiring as great force to lift the structures in the oral cavity to expose the glottis [18], suggesting that an increase in heart rate is less likely.

In studies that reported no difference in hemodynamic changes using either the Macintosh laryngoscope and AWS for endotracheal intubation, one study [6] used fentanyl ( $2 \mu \mathrm{g} / \mathrm{kg})$, thiopental (5 mg/kg), succinylcholine ( $1 \mathrm{mg} / \mathrm{kg}$ ) in inducing anesthesia while another study [7] used fentanyl $(1 \mu \mathrm{g} / \mathrm{kg})$ and propofol (1.5-2.0 mg/kg) injection and then immediately ventilated with sevoflurane ( 5 vol\%) through a mask. Our study did not use an opioidand further studies are needed to confirm whether the difference in heart rate increase between the two laryngoscopes after intubation is caused by opioid or by characteristics of severe burn patients.

Endotracheal intubation often causes damage in the airway mucosa and develops sore throat after the operation, which is one of the complaints by patients after surgery. The width of the AWS blade is $30 \mathrm{~mm}$, which is bigger than the $13 \mathrm{~mm}$ blade of the Macintosh laryngoscope [6], and the way to use the AWS is by lifting the epiglottis and applying pressure, which can cause sore throat. However, the development of sore throat after endotracheal intubation as well as the influence on the airway mucosa and the epiglottis was similar with both laryngoscopes, and the AWS can therefore be used safely. In addition, is have been suggested that smooth manipulation is possible with at least $25 \mathrm{~mm}$ distance between the upper and lower canines when opening the mouth as much as possible [19], but successful endotracheal intubation was reported with a 20 mm distance [20]. Thus, it is considered that AWS can be used in any condition in which the Macintosh laryngoscope can be used, unless left-right mouth opening is seriously limited due to perioral contracture.

In conclusion, endotracheal intubation using AWS in severe burn patients can reduce heart rate changes compared to the Macintosh laryngoscope. In addition, AWS was very useful for endotracheal intubation of severe burn patients because the degree of glottis exposure was great and the success rate of endotracheal intubation was high without pressing the thyroid cartilage or using a stylet, and the postsurgical development of sore throat was not different from other methods.

\section{References}

1. Practice guidelines for management of the difficult airway: an updated report by the American Society of Anesthesiologists task 
force on management of the difficult airway. Anesthesiology 2003; 98: 1269-77.

2. Woodson LC, Sherwood ER, Aarsland A, Talon M, Kinsky MP, Morvant EM. Anesthesia for burned patients. In: Total Burn Care. 3rd ed. Edited by Herndon DN: Philadelphia, Saunders. 2007, pp 196-228.

3. Suzuki A, Toyama Y, Katsumi N, Kunisawa T, Sasaki R, Hirota K, et al. The Pentax-AWS rigid indirect video laryngoscope: clinical assessment of performance in 320 cases. Anaesthesia 2008; 63: 641-7.

4. Enomoto Y, Asai T, Arai T, Kamishima K, Okuda Y. Pentax-AWS, a new videolaryngoscope, is more effective than the Macintosh laryngoscope for tracheal intubation in patients with restricted neck movements: a randomized comparative study. Br J Anaesth 2008; 100: 544-8.

5. Asai T, Enomoto Y, Shimizu K, Shingu K, Okuda Y. The Pentax-AWS video-laryngoscope: the first experience in one hundred patients. Anesth Analg 2008; 106: 257-9.

6. Cha SM, Kang H, Baek CW, Park JW, Jung YH. A comparative study on the efficacy and cardiovascular response generated by macintosh and pentax-AWS video laryngoscopic endotracheal intubation methods. Korean J Anesthesiol 2009; 56: 146-50.

7. Suzuki A, Toyama Y, Katsumi N, Kunisawa T, Henderson JJ, Iwasaki H. Cardiovascular responses to tracheal intubation with the Airway Scope (Pentax-AWS). J Anesth 2008; 22: 100-1.

8. Nishikawa K, Matsuoka H, Saito S. Tracheal intubation with the PENTAX-AWS (airway scope) reduces changes of hemodynamic responses and bispectral index scores compared with the Macintosh laryngoscope. J Neurosurg Anesthesiol 2009; 21: 292-6.

9. Ochroch EA, Hollander JE, Kush S, Shofer FS, Levitan RM. Assessment of laryngeal view: percentage of glottic opening score vs Cormack and Lehane grading. Can J Anaesth 1999; 46: 987-90.
10. Karkouti K, Rose DK, Wigglesworth D, Cohen MM. Predicting difficult intubation: a multivariable analysis. Can J Anaesth 2000; 47: 730-9.

11. Rose DK, Cohen MM. The incidence of airway problems depends on the definition used. Can J Anaesth 1996; 43: 30-4.

12. Adnet F, Baillard C, Borron SW, Denantes C, Lefebvre L, Galinski $\mathrm{M}$, et al. Randomized study comparing the "sniffing position" with simple head extension for laryngoscopic view in elective surgery patients. Anesthesiology 2001; 95: 836-41.

13. Rose DK, Cohen MM. The airway: problems and predictions in 18,500 patients. Can J Anaesth 1994; 41: 372-83.

14. Domino KB, Posner KL, Caplan RA, Cheney FW. Airway injury during anesthesia: a closed claims analysis. Anesthesiology 1999; 91: 1703-11.

15. Henderson JJ, Popat MT, Latto IP, Pearce AC. Difficult Airway Society guidelines for management of the unanticipated difficult intubation. Anaesthesia 2004; 59: 675-94.

16. Suzuki A, Abe N, Sasakawa T, Kunisawa T, Takahata O, Iwasaki H. Pentax-AWS (Airway Scope) and Airtraq: big difference between two similar devices. J Anesth 2008; 22: 191-2.

17. Shribman AJ, Smith G, Achola KJ. Cardiovascular and catecholamine responses to laryngoscopy with and without tracheal intubation. Br J Anaesth 1987; 59: 295-9.

18. Koyama J, Aoyama T, Kusano Y, Seguchi T, Kawagishi K, Iwashita T, et al. Description and first clinical application of AirWay Scope for tracheal intubation. J Neurosurg Anesthesiol 2006; 18: 247-50.

19. Hirabayashi Y, Seo N. Airway Scope: early clinical experience in 405 patients. J Anesth 2008; 22: 81-5.

20. Bae JY, Kim JW, Woo CH, Kim KM. The use of Pentax-AWS for difficult intubation due to postburn contracture. Korean J Anesthesiol 2008; 55: 485-9. 